\title{
Railway Scheduling System Using Repair-based Approach
}

\author{
Te-Wei Chiang and Hai-Yen Hau \\ Department of Electrical Engineering \\ National Taiwan University \\ Taipei, Taiwan 10617, R.O.C.
}

\begin{abstract}
In this paper, we propose an approach for railway scheduling based on iterative repair, a technique that starts with a complete but possibly flawed schedule and searches through the space of possible repairs. Based on the observation that the decision of the track assignments of trains would influence the performance of the resulting schedule, we divide the scheduling process into two levels. In the first level, a route preprocessing algorithm based on local search techniques is developed to find the appropriate track assignments for each train. In the second level, an iterative repair algorithm based on tabu search techniques is applied to repair the flawed schedule. The search is guided by an earliest-conflict-first heuristic that attempts to repair the earliest constraint violation while minimizing the value of objective function. We tested this approach with a randomly generated instance and found that it can work in an effective manner.
\end{abstract}

\section{Introduction}

In railway systems, the timetable containing the arrival/departure times and the track assignments of all trains at each station is the most essential schedule for day-to-day operations. Similar to conventional job-shop scheduling problem[1], the railway scheduling problem is the decision of the arrival/departure times and the assigning of resources (tracks) to all trains while minimizing a particular objective function and satisfying some specified constraints. The resources to be allocated include the tracks within a station and the tracks between two stations. For most railway systems, there are two tracks between two neighboring stations, one for southbound trains and the other for northbound trains, assuming the railway system is south-north rail. In our system, we assume the routes between stations are prespecified and only the tracks within stations need to be assigned by the scheduler. On the other hand, the decision of the arrival/departure times of all trains at each station must satisfy the specified constraints, e.g., minimum headway constraints .

An analogy can be made between the railway schedul- ing and the conventional job-shop scheduling. In railway scheduling problems, we can regard the tracks as machines in a job-shop and the assignment of trains to tracks as the dispatching of jobs to machines. From job-shop scheduling's point of view, there are two categories of machines available in railway scheduling, i.e., the tracks within stations (Type I machines) and the tracks between stations (Type II machines). For Type I machines, the processing time for an operation corresponds to the stopover time of a train at a station. For Type II machines, the processing time of an operation of a job corresponds to the running time of a train between two stations. The processing time for each operation on a Type II machine is constant (we usually assume that the running time of a train between two stations is constant since the distance between the two stations and the speed of the train are known in advance) while the processing time for operation on Type I machine can be adjusted (we have to adjust the stopover time of each train at each station the train stops at in order to avoid conflicts). The objective of railway scheduling is to minimize the average running time of each train, which corresponds to minimize the average flow time of each job in job-shop scheduling.

The main difference between the railway scheduling problem and the conventional job-shop scheduling problem lies in the constraints the final schedule is subject to. There are many conditional constraints that depend on the track assignments of each train. Moreover, we must select an appropriate machine from a set of alternative Type I machines for each operation that is processed on Type I machines. Therefore, the railway scheduling problem is similar to the conventional jobshop scheduling problem with alternative machines. Such differences make the problem more difficult than conventional job-shop scheduling problem and make it impossible to be solved by conventional OR techniques, so we resort to AI techniques.

There is a long history of AI programs that use repair or debugging strategies to solve problems. Minton et al. [11] suggested a repair method based on min-conflicts heuristic for constraint satisfaction and scheduling problem problems. Zweben et al. [13] proposed a general 


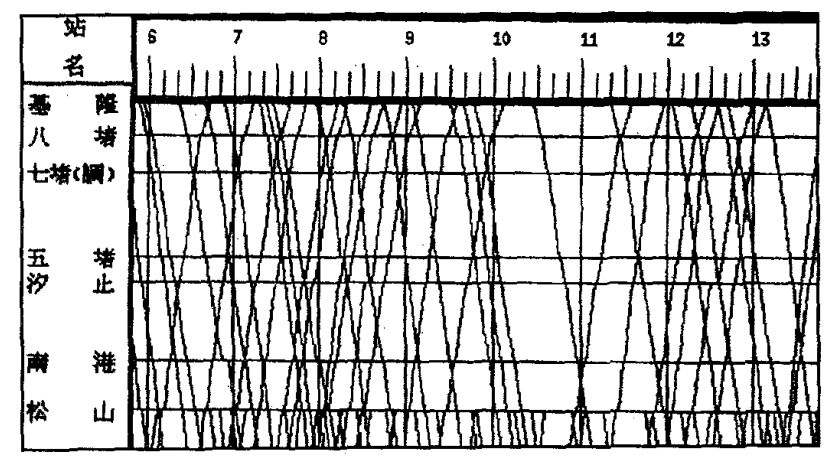

(a)

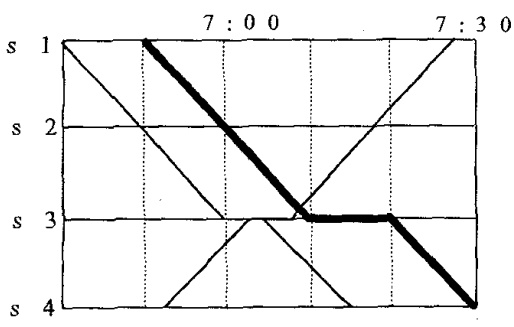

(b)

Fig. 1. (a) An example of portion of train diagram. (b) a simple train diagram used for illustration.

scheduling system being applied to the Space Shuttle ground processing problem based on iterative repair. In repair-based approach, one starts with a complete but possibly flawed schedule and searches through the space of possible repairs. The search can be guided by a repair heuristic, such as min-conflicts heuristic that attempts to minimize the number of constraint violations after each step. The heuristic can be used with a variety of different search strategies. Recently, search methods like Local Search (LS) [12], Simulated Annealing (SA) [9], and Tabu Search(TS) [5], [6], [7] have emerged. These techniques are very efficient in solving combinatorial optimization problems and have several inherent advantages, such as implementation ease and the ability to both generate solutions and improve existing ones.

Several attempts have been made in the past to tackle the complexities involved in the automatic generation of timetables [2], [3], [4], [10]. However, their railway systems usually are special cases of a general railway system. In this paper, we describe a novel railway scheduling scheme for general railway systems. There are four basic components in our system: Initial Scheduler, Repair Scheduler, Local Scheduler and Conflict Management. First, an initial train diagram is established by the Initial Scheduler according to the master scheduling plan. The Repair Scheduler uses an earliest-conflictfirst heuristic to repair conflicts sequentially after the Conflict Management finds all conflicts in the initial schedule. In local scheduling, the Local Scheduler resolves the conflict given by Repair Scheduler using one of the proposed five repair methods while minimizing the objective function. Thus the Repair Scheduler and the Local Scheduler cooperate in harmony to generate the complete schedule. During each repairing cycle, the search technique (either simulated annealing or tabu search) is used to aid the selection of appropriate repair method such that the objective function is minimized.
Moreover, in order to improve the performance of scheduling, a route preprocessing algorithm based on local search is applied to determine the appropriate initial track assignments of each train.

\section{Problem description}

Since the ultimate goal of railway company is to provide good service for passengers (e.g., the waiting time for each passenger is as short as possible) while minimizing the operation costs (e.g. each train is full load), a master scheduling plan is generated by the railway company to satisfy these requirements. In other words, we must generate a conflict-free schedule in accordance with the master scheduling plan. The master scheduling plan contains, for each train, the departure time of the starting station, the destination, and the stations along the route that the train must stop. The railway scheduling process is carried out by drawing a train diagram. The train diagram indicates, for each train, the arrival time and departure time of each station along the route, and the stopover time at a station if the train makes a stop at that station. Fig. 1 (a) shows portion of a train diagram, where the vertical axis represents stations and the horizontal axis represents time. Slanted lines represent trains. The lines with positive slope represent northbound trains and that with negative slope represent southbound trains. Fig. 1 (b) is a zoom-in version of a train diagram at station S3. The thick line in the diagram represents the southbound train that departs station $\mathrm{S} 1$ at 6:50 a.m., passes through $\mathrm{S} 2$, and arrives at $\mathrm{S} 3$ at 7:10, and stops at $\mathrm{S} 3$ for 10 minutes and departs $\mathrm{S} 3$ at 7:20. The goal of railway scheduling is to draw a train diagram for all trains and stations and determine the track assignments for each train at each station, and maximize (or minimize) certain performance criteria, subject to some physical constraints.

In this paper we assume that all trains run at the same 


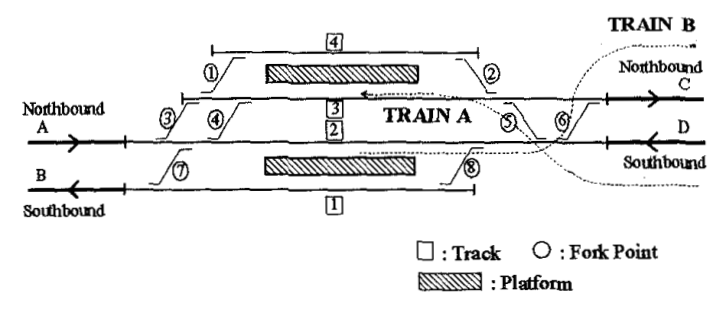

Fig. 2. A sample station configuration.

speed and there are two tracks between any two neighboring stations, one for northbound trains and the other for southbound trains.

The physical constraints to the railway scheduling are:

1) Running time constraints: These constraints indicate that the shortest running time needed for trains to travel from one station to the next station is constant.

2) Minimum stopover time constraints : The minimum stopover time of each train at each station, which is the minimum time required for passengers to get on or off the train at that station, is prespecified according to the master scheduling plan.

3) Minimum headway constraints : Due to the safety concern, there must be a safety time separation between the consecutive arrival/departure of trains at each station. These constraints specify the minimum headway between consecutive arrivals/departures at each station.

4) Level crossing constraints: The time interval between two trains sharing the same crossover must satisfy a prespecified minimum time interval to prevent collision. Note that the crossovers corresponds to the fork points shown in Fig. 2.

5) Track assignment constraints : If two trains had been allocated to the same track in a station, then there is a minimum time interval limited to the two trains.

Corresponding to these constraints are five types (Type I - Type V) of conflicts that may arise during the scheduling process, each of which corresponds to one of the constraint type defined above. For instance, Type I conflict corresponds to the violation of running time constraint. Each conflict is associated with either one train (Type I and Type II) or two trains (Type III - Type v) and the station where the conflict happens. We can find that there are many conditional constraints (e.g. (4)-(5)). The minimum headway constraints, the level crossing constraints and the track assignment constraints are route-dependent constraints, i.e., whether these constraints exist or not depends on the track assignments. Such complex constraints make the problem impossible to be solved by conventional OR techniques, not to say the inherently large scale of the problem.

In summary, the railway scheduling problem is more difficult than the job-shop scheduling problem because it is inherently large-scale and there are conditional constraints, many alternative machines (tracks), and the processing time (stopover time) may be adjustable.

\section{Proposed architecture \\ 3.1 Problem formulation}

Generally speaking, the goal of the railway scheduling is to minimize the average running time and the start time deviation of each train while satisfying all of the defined constraints. This is because we must generate a conflict-free schedule in accordance with the master scheduling plan so as to provide good service for passengers. The start time deviation of a train is the difference between the actual start time of the train and the start time planned in master scheduling plan. Mathematically, the problem can be formulated as a constrained optimization problem: the objective function is the minimization of the average total running time and the start time deviation of each train, and the constraints are that defined in section II. We can transform the constrained optimization problem to unconstrained optimization problem via the incorporation of the constraint violations into the objective function.

Assume that $\underline{x}=\left(x_{1}, x_{2}, \ldots, x_{N}\right)$ is a solution point (or a schedule) in the problem space which is composed of $x_{j}$, $j \in\{1,2, \ldots, N\}$, where $x_{j}$ corresponds to a resource(track), arrival time, departure time triplet $[r(j), a(j)$, $d(j)]$ and $N$ is the total number of $x_{j}$. Each $x_{j}$ corresponds to a stopover of a train at a station. The objective function is

$$
C(\underline{x})=P(\underline{x})+\lambda Q(\underline{x})
$$

where $P(x)$ represents the cost due to the delay of the stopover times of trains and the deviation of the departure time of the trains at their starting stations, which is our measure for schedule quality; $Q(\underline{x})$ is the cost due to the conflicts in schedule $\underline{x}$ and $\lambda$ is the Lagrange multiplier used to relax the constraint violations. We usually refer the objective function $C(x)$ to the cost function of the problem. For simplicity, we call $C(\underline{x}), P(\underline{x})$ and $Q(\underline{x})$ the total cost, the conflict cost and the schedule cost of the schedule respectively.

Since the minimization of average total running time is equivalent to the minimization of the summation of all stopover times and total start time deviation is equivalent to the start time deviation of each train, the $P(\underline{x})$ can be defined as $\Sigma_{j} p_{j}$, where $p_{j}$ is the cost of the schedule of operation $x_{j}$ and is defined as 


$$
p_{j}=\alpha|d(j)-a(j)|+\beta\left|d(j)-d_{0}(j)\right| \Delta_{j}
$$

where $d_{0}(j)$ represents the ideal departure time of the train at its starting station, which is obtained from the master scheduling plan. $\Delta_{j}$ is equal to 1 if $x_{j}$ corresponds to the operation of the associated train at its starting station and is equal to 0 otherwise. The $\alpha$ and $\beta$ represent the weight of the delay time and the weight of the start time deviation. On the other hand, $Q(\underline{x})$ can be defined as $\Sigma_{k} q_{k}$, where $q_{k}$ is an positive integer representing the time interval the $k$ th conflict is violated, assuming there are $M$ conflicts in the schedule $\underline{x}$. The weight $\alpha, \beta$ and Lagrange multiplier $\lambda$ are chosen to satisfy $\alpha<<\lambda$ and $\beta<<\lambda$ since a conflict-free schedule is our ultimate goal.

In fact, the objective function automatically follows the philosophy of min-conflicts heuristic since the direction of the decrease of the objective function is the same as the direction of the minimization of conflicts. In a word, the underlying concept of the objective function is to generate a conflict-free schedule while minimizing the total running time and the start time deviation of each train.

\subsection{System architecture}

The four basic components in the proposed system are : Initial Scheduler, Repair Scheduler, Local Scheduler and Conflict Management. First, an initial train diagram is established by Initial Scheduler according to the master scheduling plan. The track assignments of each train at each station are randomly assigned. Since the initial schedule is established regardless of constraint violations, there are many conflicts in the initial schedule. The Repair Scheduler then determines the repairing sequence of conflicts after Conflict Management find all conflicts in the initial schedule. The sequence of conflicts to be repaired in the proposed system is based on the earliestconflict-first heuristic that the earliest constraint violation, which corresponds to the leftmost conflict in the train diagram, will be solved first by the Local Scheduler. From local search's point of view, the neighborhood of the current solution point is the set of solution points after all possible repairs of the earliest conflict in the current solution point. Since the leftmost conflict in the train diagram will be solved first, there exists a boundary whose left side is conflict-free. As shown in Fig. 3, thick line indicates the boundary of the conflict-free area, hollow circles indicate conflicts, and the solid circle represents the earliest conflict. During each repairing cycle, the earliest conflict will be solved using the repair method selected by either simulated annealing or tabu search. Through the appropriate selection of the repair

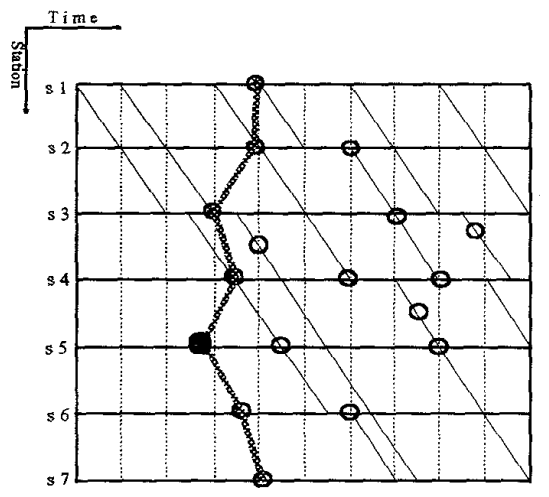

Fig.3. Illustration of the earliest-conflict-first heuristic.

methods (i.e., limit the use of repair methods that can cause the left movement of the boundary), the boundary will move to right gradually until the overall train diagram is conflict-free. From another point of view, we can look upon the initial train diagram as a reference schedule that guides the Local Scheduler. In local scheduling, the conflict given by Repair Scheduler is repaired in the direction of minimizing the objective function. As such, two schedulers, the Repair Scheduler and the Local Scheduler, cooperate in harmony to generate the complete schedule.

\section{Proposed algorithm}

In the section we will first introduce how to repair a conflict by some repair methods and then propose some algorithms to repair all conflicts generated by Initial Scheduler.

\subsection{Repair methods}

Recall that each conflict is associated with either one train (Type I and Type II) or two trains (Type III - Type VII) and the station where the conflict happens. When a conflict arises between two trains at some station, one of the trains will be selected in an attempt to repair as much of the reduction of the cost function as possible. The system will try to move the train left or right in time axis so that the resource is available, rather than exploring many possible alternatives.

There are five repair methods that can repair a conflict: 1) Change the track assignment of a train at the station.

2) Left-shift the stopover time of the violated train at the station in the train diagram such that the constraint is satisfied. Notice that although the stopover time of the train at the station and the running times of the train between any two neighboring stations do not change, this movement will reduce the stopover 
time of the train at the previous stopover station and will extend the stopover time of the train at the next stopover station. In other words, this movement only affects the stopover time of the train at the station and those at the two adjacent stations.

3) Right-shift the stopover time of the violated train at the station in the train diagram such that the constraint is satisfied. Similarly, this movement will extend the stopover time of the train at the previous stopover station and will reduce the stopover time of the train at the next stopover station.

4) Left-extend the stopover time of the train at the station (i.e. extend the stopover time while fixing the departure time of the train at this station).

5) Right-extend the stopover time of the train at the station (i.e. extend the stopover time while fixing the arrival time of the train at this station).

The first three repair methods are used to solve all types of conflicts except minimum stopover time violations. Among the three methods, the change of the track assignment has the highest priority since good track assignment can avoid some route-dependent conflicts. Furthermore, the priority of a left-shift is higher than that of a right-shift because moving train right on time axis will cause the train to occupy the resource longer and hence affect the performance of the schedule. The last two repair methods are used only for resolving minimum stopover time violation. Similarly, the priority of the left-extend stopover time is higher than right-extend stopover time. Notice that the five repair methods only adjust the stopover time of each train, so the time required for each train running between any two neighboring stations is unchanged. In other words, the Type I conflict (running time constraint violation) would not arise during the scheduling process. In addition, since the Left-shift and Left-extend repair methods may create conflicts left of the conflict-free boundary, the two repair methods must be carefully used to assure that the boundary is gradually move to right.

\subsection{Iterative repair}

To begin with, we establish the initial train diagram according to the master scheduling plan and randomly assign tracks to trains. Then the corresponding value, $c_{o}$, of the cost function is obtained. Since the initial schedule is not conflict-free, we must repair the conflicts in the initial schedule.

The repairing process is an iterative local optimization process. During each iteration, we cyclically search a repair that resolves the conflict given by the earliestconflict-first heuristic and minimizes the cost function at the same time. To select an appropriate repair method
Step 1. (Initialization)

1.1 Generate the initial schedule according to master scheduling plan.

1.2 Randomly assign tracks to train.

1.3 Find all conflicts in the initial schedule and put these conflicts to $S_{C}$.

1.4 Evaluate $c_{n}$.

1.5 count $:=0$.

Step 2. 2.1 If $S_{C}$ is empty or count $>$ limit then stop, else select and delete the earliest conflict from $S_{c}$.

2.2 Put all possible repair methods to $S_{R}$.

Step 3. 3.1 Select and delete the highest priority repair method from $S_{R}$.

3.2 Test to repair the selected conflict.

3.3 Evaluate $c_{n}$.

Step 4. If $c_{n}<c_{0}$ or $S_{R}$ is empty, then perform the repair and goto step 5 , else goto step 3 .

Step 5. 5.1 Update $S_{C}$.

$5.2 c_{o}:=c_{n}$

5.3 Increase count by one.

5.4 Goto step 2.

Fig. 4. The iterative repair algorithm base on local search techniques.

for a conflict, two basic techniques can be used, i.e., local search and simulated annealing.

4.2.1 Local Search. If a repair reduces the cost function, i.e. the new cost value, $c_{n}$, is smaller than $c_{o}$, then we accept the repair and assign $c_{n}$ to $c_{o}$; otherwise we try next priority repair until all possible repairs to the conflict has been tried. If no repair method can reduce the cost function, the lowest priority repair method (rightshift or right-extend the stopover time) will be selected to repair the conflict. We hope that in the train diagram the left-side of the repaired conflict remains conflict-free such that the searching process is well controlled. The local search algorithm is shown in Fig. 4, in which the following notations are used :

$S_{C}$ : The set of conflicts corresponding to current schedule

$S_{R}$ : The set of repair methods

$c_{o}$ : The cost of the original schedule

$c_{n}$ : The cost of the new generated schedule

count : the number of iterations

limit : a prespecified number used to limit the number of iterations in the iterative repairing process.

The local search algorithms has a tendency of getting stuck at a local optimum or a cycle. For example, there is a conflict between two trains at some station, the 
earlier train has been shifted earlier to repair the conflict. But the shift results in another new conflict. To repair the new conflict, the train has to shift later and hence the original conflict comes back. Such that a cycle occurs between the two repairs.

4.2.2 Simulated Annealing. To prevent from getting stuck at a locally minimum point, an escape function is applied. The algorithm sometimes accepts a new solution that is worse than the current solution to escape local optimum and cycles. This stochastic technique is referred to as Simulated Annealing (SA) [9]. The escape function for accepting inferior solutions is : $E\left(c_{n}, c_{\alpha}, T\right)=$ $e^{-\left|c_{o}-c_{n}\right| / T}$, where $T$ is a "temperature" parameter that is gradually reduced (i.e. cooled during the search process). When a random number between 0 and 1 exceeds the value of the escape function, the system accepts the worse solution. Note that escape becomes less probable as the temperature is lowered. The algorithm is the same as the iterative repair algorithm in Fig. 4 except that the step 4 and step 5 are modified as follows :

Step 4. If $c_{n}<c_{o}$ or $r_{i}>\mathrm{E}\left(c_{n}, c_{o}, T\right)$ or $S_{R}$ is empty, then perform the repair and goto step 5 , else goto step 3.

Step 5. 5.1 Update $S_{C}$.

$5.2 c_{o}:=c_{n}$. 5.3 if $T>T_{\min }$ then $T:=\gamma^{*} T$, else $T:=T_{m i n}$ 5.4 Increase count by one.

5.5 Goto step 2.

where the following notations are used:

$r_{i}$ : The random number between 0 and 1 which generated in $i$ th iteration

$T$ : The temperature used for escape function $E$

$T_{\min }$ : The lowest temperature used for escape function $E$

$\gamma$ : The parameter used for update temperature $T$

$E\left(c_{n}, c_{o}, T\right)$ : The escape function for accepting inferior solution which is depend on $c_{n}$ and $c_{o}$ and temperature $T$.

When we use the simulated annealing method to select a repair method, it may cause the left movement of the conflict-free boundary. This is because the creation of conflicts in the left of the boundary usually increases the value of the cost function, which is acceptable for the simulated annealing method. The phenomenon of the left movement of the conflict-free boundary would gradually reduce as the temperature cools off and the probability of accepting inferior solution decreases. Although simulated annealing can escape from locally minimum, it could be potentially time-consuming when applied to complex problem instances. Besides, it is not easy to find the optimum annealing schedule for the given problem. The initial temperature and the decrement ratio of the temperature in the annealing schedule would largely inference the performance.

4.2.3 Tabu Search. To prevent cycles, we incorporate a forbidden list into the standard local search algorithm. The forbidden list maintains a memory of cost values recently happened. When two schedules have the same cost values, we can recognize that cycle may occur since different schedules usually correspond to different cost values. Because cycle usually generated from the interactions of the left-shift/left-extend repairs and the rightshift/right-extend repairs, when a cycle occurs, we disable the left-shift/left-extend repairs to escape the cycle.

\subsection{Route preprocessing}

Based on the observation that the route-dependent constraints depend on the track assignments of each train, we only apply the first repair method (track reassignment) and local search to increase the fitness of the track assignments prior to the iterative repairing process, which we called route preprocessing. During route preprocessing, for each route-dependent constraint violation (described in Section III), the track assignments of the associated trains at the conflicting station will be changed if the change can reduce the value of the cost function. Note that in our implementation the conflicts are automatically maintained by the Conflict Management, so we can easily obtain the conflict information about the current schedule after each repair iteration. After route preprocessing, the assignments of tracks with lower cost value will be found, and the following iterative repairing process will be more efficient and effective.

\section{Experimental results}

In our experiments, we set $\alpha, \beta$ and $\lambda$ in eq. (1)-(2) to 1,2 and 10 respectively. The station configuration used is the example station shown in Fig. 2. All experiments were run on a PC 80486-33. Each experiment ran until the resulting schedule was conflict-free. Since the repair functions are probabilistic, we calculated average results over five repeated trials for each experiment. In the following we present the results of these experiments.

Example 1: In Fig. 5 we graph the average cost as a function of iteration for a random generated 10-station 100 -train problem solving by simulated annealing in which the weight of conflict cost is set to 10 and the schedule range is 12 hours. The three curves in this figure represent the cost due to conflicts, the cost due to schedule performance and the total cost of the schedule (see eq. (1)) respectively. Each iteration corresponds to a repairing cycle. The initial and final temperature used 
for simulated annealing are set to 980 and 25 respectively. Besides, we let the temperature for the $i$ th iteration was : $T_{i}=0.95 T_{i-1}$. As a result, the number of iterations to achieve a conflict-free solution is 4245 for the one without route preprocessing and 4382 for the one with route preprocessing. For the one with route preprocessing, about 550 iterations of 4382 iterations are used for route preprocessing, which is equal to the number of route-dependent conflicts in the initial schedule. During route preprocessing, only track assignments of each train can be changed, so the cost due to schedule performance remains unchanged (see Fig. 5(b)). The average total cost is $\mathbf{4 0 3 . 8}$ for the one without route preprocessing and 390 for the one with route preprocessing.

Example 2: In Fig. 6 we graph the average cost as a function of iteration for the same problem as that of example 1 solving by tabu search. We can find that, in Fig. 6 (a), the conflict cost is decreasing during the repairing process and achieve zero (i.e. conflict-free) after about 1100 iterations. As a result, the average number of iterations to achieve a conflict-free solution is 1149 for the one without route preprocessing and 1524 (about 550 of which are used for route preprocessing) for the one with route preprocessing. The average total cost is 326 for the one without route preprocessing and 239.4 for the one with route preprocessing.

\section{Conclusions}

The railway scheduling problem is more difficult than conventional job-shop scheduling problems because it is inherently large-scale and there are conditional constraints, many alternative machines (tracks), and the processing time (stopover time) may be adjustable. In this paper, we demonstrated a railway scheduling system based on the iterative repair method. We introduced how to transform the railway scheduling problem into a repair-based search problem. Searching through the space of possible repairs, the system can quickly find a good feasible schedule without trying all possible alternatives. In addition, the proposed earliest-conflict-first heuristic can generate a boundary whose left side is conflict-free. Through the cooperation of the heuristic and the search methods, such as simulated annealing and tabu search, the boundary will gradually move to right and eventually result in a conflict-free schedule. Moreover, the concept of route preprocessing is proposed to find the good track assignments in advance, which can conquer the difficulty resulting from the conditional constraints and largely improve the performance of the resulting schedule. Experimental results show that an iterative repair algorithm based on $t a b u$ search performs better than that based on simulated annealing in both of the convergence speed and the schedule quality. In conclusion, the proposed repair-based system can resolve the large-scaled railway scheduling problem in an efficient and effective manner.

\section{References}

[1] K. Baker, "Introduction to sequencing and scheduling," John Wiley \& Sons, 1974.

[2] T. W. Chiang and H. Y. Hau, "Knowledge-based technology for railway scheduling," in Proc. Int. Conf. on Industrial Fuzzy Control Intelligent Systems, Houston, Texas, pp. 42-46, 1993.

[3] J. E. Cury, F. A. C. Gomide, and M. J. Mendes, "A methodology for generation of optimal schedules for an underground railway system," IEEE Trans. on Automatic Control, vol. AC-25, no. 2, April 1980.

[4] K. Fukumori, H. Sano, "Fundamental algorithm for train scheduling based on artificial intelligence," Systems and Computers in Japan, vol. 18, no. 3, 1987.

[5] F. Glover, "Tabu search - part I," ORSA J. Computing, vol. 1, pp. 190-206, 1989.

[6] F. Glover, "Tabu search - part II," ORSA J. Computing, vol. 2, pp. 4-32, 1990.

[7] F. Glover, E. Taillard, and D. Werra, "A user's guide to tabu search," Annals of Operations Research, vol. 41, pp. 3-28, 1993.

[8] J. Gu, "Local search for satisfiability (SAT) problem," IEEE Trans. on Systems, Man, and Cybernetics, vol. 23, no. 4, pp. 1108-1129, 1993.

[9] S. Kirkpatrick, C. D. Gelatt, and M. P. Vecchi, "Optimization by simulated annealing," Science, vol. 220, no. 4598, pp. 671-680, 1983.

[10] K. Komaya and T. Fukuda, "A Knowledge-based approach for railway scheduling," in Proc. CAIA, pp.405-411, 1991.

[11] S. Minton, M. D. Johnston, A. B. Philips, and P. Laird, "Minimizing conflicts: a heuristic repair method for constraint satisfaction and scheduling problems," Artificial Intelligence, vol. 58, pp. 161-205, 1992.

[12] R. Sosic and J. Gu, "Efficient local search with conflict minimization : A case study of the $n$-queens problem problem, IEEE Trans. on Knowledge and Data Engineering, vol. 6, no. 5, pp. 661-668, 1994.

[13] M. Zweben, E. Davis, B. Daun, and M. J. Deale, "Scheduling and rescheduling with iterative repair," IEEE Trans. on Systems, Man, and Cybernetics, vol. 23 , no. 6, pp. 1588-1596, 1993. 


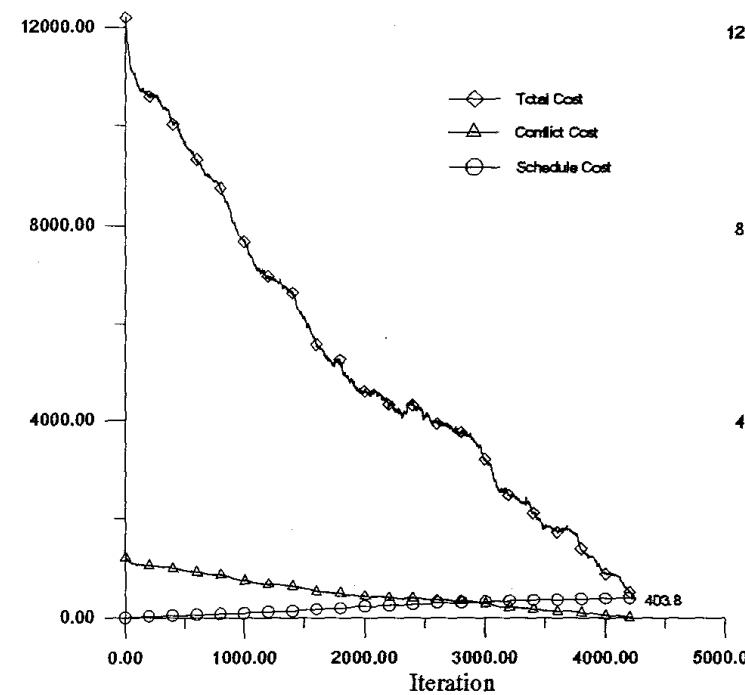

(a)

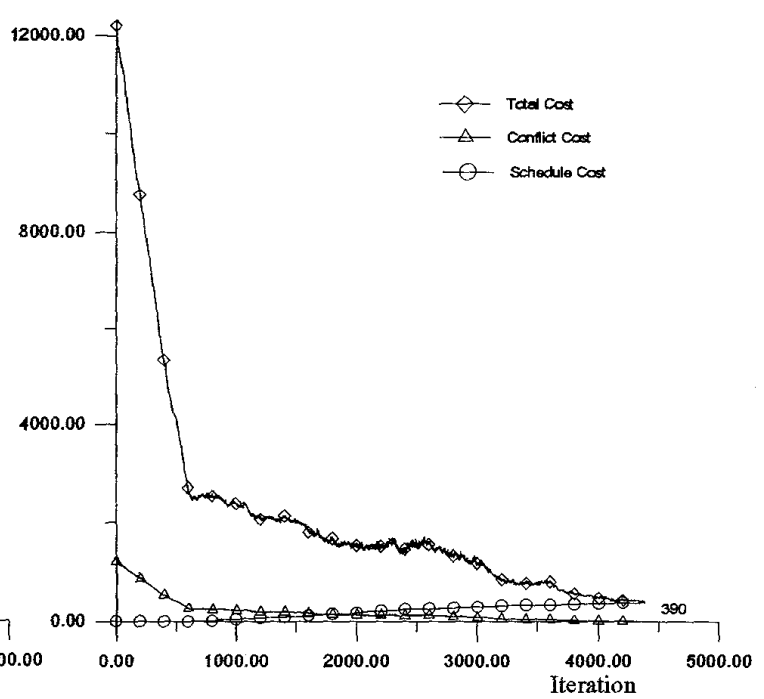

(b)

Fig. 5. Results of scheduling a randomly generated 10-station 100-train problem using simulated annealing. (a) without route preprocessing. (b) with route preprocessing.

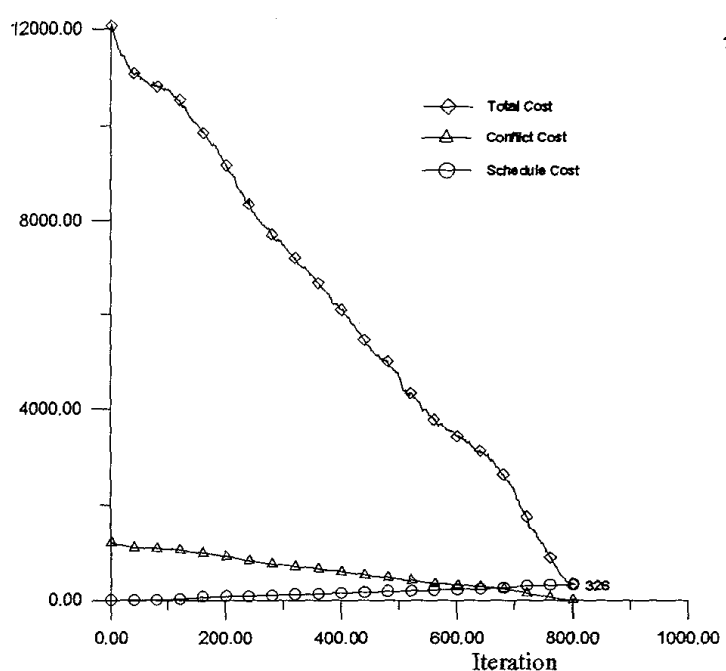

(a)

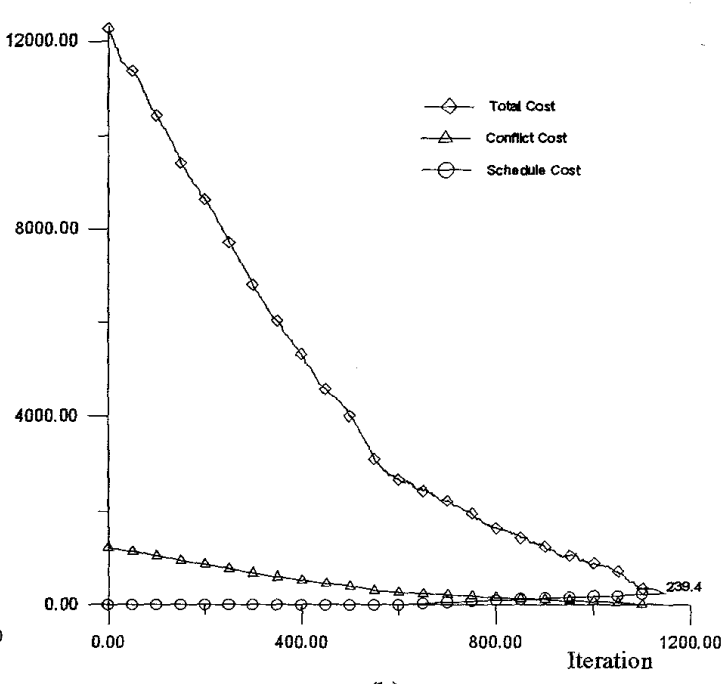

(b)

Fig. 6. Results of scheduling a randomly generated 10-station 100-train problem using tabu search. (a) without route preprocessing. (b) with route preprocessing. 